\section{Long-term outcomes after discharge}

\section{from medium secure care: a cause for concern}

STEFFAN DAVIES, MARTIN CLARKE, CLIVE HOLLIN and CONOR DUGGAN

\begin{abstract}
Background There are few long-term follow-up studies of patients discharged from medium secure units in the UK, even though these units were introduced over 20 years ago.
\end{abstract}

Aims To describe mortality, rates of reconviction at different time periods; violent behaviour (not leading to conviction), readmission and employment, after discharge from a medium secure unit.

Method Of 595 first admissions over a 20-year period, 550 discharged cases were followed-up. Multiple data sources were used.

Results Fifty-seven (10\%) patients had died, of whom 18 (32\%) died by suicide, and the risk of death was six times greater than in the general population. Almost half (49\%) of those discharged were reconvicted and almost two-fifths (38\%) of patients were readmitted to secure care.

Conclusions Community psychiatric services need to be aware that those discharged from medium secure care are a highly vulnerable group requiring careful follow-up if excess mortality, high levels of psychiatric morbidity and further offending are to be prevented.

Declaration of interest None. Funding detailed in Acknowledgements.
Although the psychiatric management of specific individuals has often had a disproportionate effect on mental health policy in the UK, data on the outcome of the generality of patients from secure psychiatric services are surprisingly limited, although these are low-volume and high-cost forms of care. Medium secure services are a case in point, with few data whereby their efficacy can be judged. In one of the few published studies, Maden et al (1999) found that in $24 \%$ of 234 first admissions the patient had been reconvicted at a mean of 6.6 years, $9 \%$ of patients had died and $74 \%$ had been readmitted. Reconviction in a larger sample $(n=959)$ followed up for 2 years was $15 \%$ (Maden et al, 2004). Given the limited nature of previous work, we sought to extend it in this study by examining the fate of an entire first admission cohort to medium security across a range of outcomes and services, over a 20-year period, using multiple sources of information.

\section{METHOD}

\section{Sample}

The study sample comprised all admissions to the Arnold Lodge medium secure unit in Leicester since its opening in July 1983 up to 30 June 2003 - a period of 20 years. Ricketts et al (2001) previously reported on the characteristics of the 504 patients admitted to this unit up to 30 June 1999. Those data were checked again as part of this study and a further 8 patients were identified, together with an additional 83 patients who were admitted between 1 July 1999 and 30 June 2003. Thus, there were in total 595 first admissions to Arnold Lodge over the 20 years since the unit opened.

\section{Data sources}

Admission characteristics were derived from medical records at Arnold Lodge. Outcome data were obtained from clinical records at Arnold Lodge, other psychiatric services, the Home Office Mental Health Unit, the Office for National Statistics (ONS), the general practitioner registrations database, the Offenders' Index and the Police National Computer (for reconvictions), the electoral roll (UK-Info Disk version 10, i-CD Publishing, London, UK) and the LexisNexis database of newspaper reports.

\section{Outcome measures}

A proforma was designed to record several outcomes, including data on reconviction, psychiatric contact, accommodation and psychosocial variables for each year of follow-up for each case in the study. In this paper we report on the sample's mortality, reconviction, behaviours not resulting in conviction, readmission to secure and open hospitals, employment and accommodation.

\section{Mortality}

Death certificates were obtained from the ONS. The mortality of the sample overall was compared with that of the general population by indirect standardisation to the England and Wales mortality rates for 10-year age bands published by the ONS (http://www.statistics.gov.uk). This yielded a standardised mortality ratio together with its $95 \%$ confidence interval.

\section{Classifying reconvictions}

Although over 30 forensic follow-up studies have been published to date, none has used the Home Office standard method for reporting reconviction. This classifies convictions as either 'grave' or 'standard list' offences (Home Office, 2002). Grave offences are those for which the maximum sentence is life imprisonment, plus arson not endangering life, and include murder, attempted murder, robbery, rape and arson. Standard list offences are all other indictable offences tried in either a Crown court or a magistrate's court. The Home Office also standardises its reporting of reconviction, for example at 2 years and 5 years following the date of release from prison. The authors have adopted this classification because it allows comparison with criminal justice statistics.

In line with Home Office practice, the date of the conviction rather than the actual date of the offence was used in the time to reconviction analyses because the date of the actual offence was not available for all 
offences, although it is accepted that this is a conservative approach that is likely to underestimate the rate of reoffending. The Police National Computer database provides the date when a person was charged, but this information was not available in all cases. The time to reconviction presented in the study was calculated from the point of discharge from Arnold Lodge rather than time of entering the community. However, where the case notes or reports were available, violent and aggressive episodes and fire-setting were recorded including those by patients in hospitals, prison or the community.

\section{Ethical considerations}

Ethical approval was granted from the Trent Multicentre Research Ethics Committee. In the light of the known difficulty in both identifying and gaining the consent of forensic psychiatric patients, the research was conducted under section 60 of the Health and Social Care Act 2001. This permits the use of identifiable National Health Service (NHS) patient information under certain circumstances, without the consent of patients. This was the first study of a psychiatric population to be granted section 60 approval. Statistical analyses were conducted using the Statistical Package for the Social Sciences, version 11.5 for Windows.

\section{RESULTS}

The catchment area originally served by the Arnold Lodge unit was the former Trent Region comprising the counties of Leicestershire, Nottinghamshire, Derbyshire, Lincolnshire and South Yorkshire (population approximately 4.8 million according to ONS estimates for mid-2001). However, this area was reduced in 1997 - 14 years into the follow-up - to Leicestershire, Nottinghamshire, South Derbyshire and Lincolnshire (approximate population 3.3 million) when another medium secure unit opened in the north of the region.

Of the 595 first admissions, there were 502 men $(84.4 \%)$ and 93 women $(15.6 \%)$. Four people had died during their admission, 550 people had been discharged and 41 people had yet to be discharged at the census date. Hence, 554 'discharges' constituted the sample used in the analyses (apart from mortality, which included all admissions). The mean length of stay for this sample was 346 days (s.d. $=468.2)$, ranging from 2 days to 3872 days. The mean age on admission was 29.9 years (s.d.=9.1). The Mental Health Act 1983 classification of these admissions comprised $67.2 \%$ mental illness, $26.6 \%$ psychopathic disorder, $3.0 \%$ mental illness and psychopathic disorder, and $0.5 \%$ mental impairment; the classification for $2.4 \%$ was unknown and $0.3 \%$ did not have a classification.

The mean length of follow-up from discharge to death, loss of contact, or the census date was 9.4 years (s.d.=4.8). Women had a longer mean follow-up time than men -11.5 years $($ s.d. $=4.1)$ and 9.0 years (s.d. $=4.8) \quad$ respectively $(t=5.062$, d.f. $=$ 144.9, $P<0.001)$. There was no significant difference in the mean follow-up times between patients with a Mental Health Act classification of either psychopathic disorder (9.8 years, s.d. $=5.1$ ) or mental illness (9.2 years, s.d. $=4.6)$. Overall there were 5771 person-years of follow-up from admission (including mortality on the unit) and 5246 person-years from discharge.

\section{Discharge location}

Of the 554 'discharges', $34.3 \%$ were discharged to a psychiatric hospital of a lower security, predominantly open wards; $27.3 \%$ were discharged to the community (which includes home or a hostel); $26.5 \%$ of patients were transferred to the criminal justice system, either returned to prison or to court for sentencing; $7.2 \%$ were transferred to high secure care; $2.9 \%$ were transferred to a different medium secure unit; $0.7 \%$ died while in the unit; and the discharge location was unknown for $1.1 \%$, mainly due to their being discharged in their absence after going absent without leave or failing to return from leave.

\section{Mortality}

At the census, whether the individual was alive or dead was known for 522 of the 550 discharged patients $(95 \%)$. However, 23 of the remaining 28 patients were confirmed as being alive to at least 2000 (data from electoral rolls, general practitioner registrations and the Police National Computer). Four patients died during their first admission and a further 53 patients died after discharge. The crude risk of death was $9.6 \%$ overall $(9.2 \%$ for men and $14.6 \%$ for women) with a mean age at death of 43.6 years (s.d.=12.9). Only 25 deaths $(44 \%)$ had a verdict of natural causes. There were 18 deaths from suicide
$(32 \%)$ and $14(25 \%)$ from other unnatural causes (Table 1).

Standardised mortality ratios (SMRs) were calculated from admission rather than discharge so as to include the four individuals who died in hospital during their admission. The risks of death for men, women, the whole cohort and deaths by different causes were all significantly higher than those expected in the general population (Table 2). For instance, the risk of death was 6 times greater than expected for the whole cohort, almost 19 times greater for deaths from unnatural causes and over 32 times greater for deaths from suicide. The SMRs for the Mental Health Act legal classifications of mental illness and psychopathic disorder were 6.3 and 4.6 respectively.

\section{Reconviction}

Almost half $(48.7 \%)$ of those discharged were reconvicted of an offence over the entire period of follow-up (264 of 542) reconviction data for 8 patients were

Table I Cause of death

\begin{tabular}{ll}
\hline Cause of death & $n$ \\
\hline Natural & \\
Cancer & 5 \\
Bronchopneumonia & 6 \\
Heart-related & 9 \\
Other (e.g. obesity, peritonitis) & 5 \\
Suicide & \\
Hanging & 10 \\
Drowning & 3 \\
Overdose/poisoning & 3 \\
Other (e.g. fall from height) & 2 \\
Open verdict & \\
Hanging & 3 \\
Collision with train & 1 \\
Fall from building & 1 \\
Poisoning & 1 \\
Collapse after medication & 1 \\
Organ failure/peritonitis & 1 \\
Misadventure & \\
Overdose/poisoning & \\
Swallowed foreign body & \\
Accidental & \\
Fall & \\
Unknown & \\
Head injuries & \\
Total & \\
\hline
\end{tabular}


Table 2 Standardised mortality ratios for the 595 admissions

\begin{tabular}{|c|c|c|c|}
\hline & \multicolumn{2}{|c|}{ Deaths } & \multirow[t]{2}{*}{ SMR $(95 \% \mathrm{Cl})$} \\
\hline & Observed & Expected & \\
\hline Total & 57 & 9.5 & $600(454-777)$ \\
\hline \multicolumn{4}{|l|}{ Gender } \\
\hline Men & 44 & 9.3 & 475 (345-638) \\
\hline Women & 13 & 1.3 & 975 (519-I668) \\
\hline \multicolumn{4}{|l|}{ MHA classification } \\
\hline Mental illness & 44 & 7.0 & 625 (454-839) \\
\hline Psychopathic disorder & 9 & 2.0 & $460(211-874)$ \\
\hline \multicolumn{4}{|l|}{ Cause of death } \\
\hline Natural & 24 & 7.8 & $309(198-460)$ \\
\hline Unnatural & 33 & 1.7 & $1898(1354-2733)$ \\
\hline Suicide & 18 & 0.6 & 3231 (1915-5107) \\
\hline
\end{tabular}

MHA, Mental Health Act; SMR, Standardised mortality ratio.

missing). The locations where the offences were committed were as follows: community $225(85 \%)$, hospital $24(9 \%)$ and prison $5(2 \%)$; the location was not known for $10(4 \%)$. The mean time from discharge to first conviction was 3.2 years (s.d. $=3.2$, median=1.9); the maximum time to conviction after discharge was 16.4 years.

Table 3 shows the number of patients who were convicted of a standard list or grave offence at 2 years, at 5 years and at any point during the follow-up. Patients without a conviction but who did not have a full 2-year or 5-year follow-up were excluded. The only significant difference for reconviction according to gender showed that men were more likely than women to have been convicted of a standard list offence in the first 5 years after discharge $\left(\chi^{2}=7.0\right.$, d.f. $\left.=1, P=0.008\right)$. The only significant difference for reconviction by Mental
Health Act classification of psychopathic disorder or mental illness showed that patients with a classification of psychopathic disorder were more likely to have been convicted of a standard list offence at any time after discharge $\left(\chi^{2}=4.5\right.$, d.f. $=1$, $P=0.034)$.

\section{Violent behaviour and fire-setting after discharge}

For various reasons not every offence leads to a conviction - particularly when patients are detained in healthcare settings. Within the first 2 years after discharge at least $28 \%$ of patients had exhibited violent behaviour not resulting in conviction and within 5 years this had increased to at least $42 \%$. Within the first 2 years after discharge at least $3 \%$ of patients had engaged in firesetting not resulting in conviction; within 5 years this had increased to at least $6 \%$.

\section{Readmission after discharge}

The majority of patients in the sample were admitted to a psychiatric hospital at some point in the follow-up. Of the 151 patients who were discharged directly to the community, $40(26 \%)$ were readmitted to a medium secure unit during the follow-up. A further 152 patients $(27.6 \%)$ who were discharged to a hospital remained in hospital for the whole of the first year after discharge, including 40 patients transferred to high secure hospitals. Overall $20.5 \%$ were readmitted to Arnold Lodge, $7.8 \%$ to other medium secure units and $14.9 \%$ to a high security hospital, with 207 of the 550 discharged $(37.6 \%)$ subsequently spending some time in medium or high security and with some patients spending time in both. Over the whole study period there were missing readmission data for 60 patients; excluding these, only 151 patients $(30.8 \%)$ were never readmitted to a psychiatric hospital.

\section{Employment}

On admission, $12.3 \%$ of patients had never been employed, and the majority $(51.3 \%)$ had been employed in unskilled jobs. Stable employment after discharge was uncommon $(14.5 \%)$ and was often provided by family members. Patients with a Mental Health Act classification of psychopathic disorder were more likely than patients with a classification of mental illness to have gained employment at some point during the follow-up period. This approached significance $\left(\chi^{2}=3.7\right.$, d.f. $\left.=1, P=0.054\right)$.

\section{DISCUSSION}

Medium secure units were introduced in the UK in the early 1980 s to fill a perceived

Table 3 Percentage of patients reconvicted of a standard list or a grave offence at 2 years and 5 years after discharge or at any time during the follow-up period

\begin{tabular}{|c|c|c|c|c|c|c|}
\hline & \multicolumn{3}{|c|}{ Standard list offence } & \multicolumn{3}{|c|}{ Grave offence } \\
\hline & 2 years (\%) & 5 years $(\%)$ & Ever (\%) & 2 years (\%) & 5 years $(\%)$ & Ever (\%) \\
\hline Men & $110 / 415(26.5)$ & $164 / 367(44.7)$ & $223 / 453(49.2)$ & $28 / 415(6.7)$ & 43/367 (II.7) & $63 / 453$ (13.9) \\
\hline Mental illness & $73 / 297(24.6)$ & $\mathrm{III} / 264(42.0)$ & $148 / 320(46.3)$ & $19 / 297(6.4)$ & $30 / 264$ (II.4) & $45 / 320(14.1)$ \\
\hline Psychopathic disorder & $28 / 93(30.1)$ & $44 / 84(52.4)$ & $61 / 107(57.0)$ & $7 / 93(7.5)$ & 10/84 (II.9) & $13 / 107(12.1)$ \\
\hline Women & $19 / 89(21.3)$ & $25 / 86(29.1)$ & $36 / 89(40.4)$ & $6 / 89(6.7)$ & $10 / 86(11.6)$ & $15 / 89(16.9)$ \\
\hline Mental illness & $5 / 44(11.4)$ & $9 / 42(21.4)$ & I5/44 (34.I) & $0 / 44(0.0)$ & $2 / 42(4.8)$ & $5 / 44(11.4)$ \\
\hline Psychopathic disorder & $13 / 40(32.5)$ & $15 / 39(38.5)$ & $20 / 40(50.0)$ & $5 / 40(12.5)$ & $7 / 39(17.9)$ & $9 / 40(22.5)$ \\
\hline All & $129 / 504(25.6)$ & $189 / 453(41.7)$ & $259 / 542(47.8)$ & $34 / 504(6.7)$ & $53 / 453$ (II.7) & $78 / 542(14.4)$ \\
\hline Mental illness & $78 / 341$ (22.9) & $120 / 306(39.2)$ & $163 / 364(44.8)$ & |9/34| (5.6) & $32 / 306(10.5)$ & $50 / 364(13.7)$ \\
\hline Psychopathic disorder & $4 I / 133(30.8)$ & $59 / 123(48.0)$ & $8 \mathrm{I} / 147(55.1)$ & $12 / 133(9.0)$ & $17 / 123(13.8)$ & $22 / 147(15.0)$ \\
\hline
\end{tabular}


gap between local services and high secure care. Arnold Lodge was one of the first of these units to open, so an investigation of the course and outcome of its patients offers a unique opportunity to examine the long-term effectiveness of these expensive facilities. We found that after discharge the outcome for patients was poor, with a mortality rate that was six times that which one might expect; that almost half of those discharged had at least one reconviction; that almost two-thirds were readmitted within 5 years after discharge; and that their capacity to obtain and retain gainful employment was very limited. Subject to certain limitations that we shall now consider, these results ought to be a major cause for concern.

\section{Limitations}

The first major limitation was that this study examined the outcome from a single unit; with its unique policies and therapeutic ethos; hence its results may not be generalisable to all medium secure units. What is needed now is similar reports from other medium secure units with which these findings can be compared. The other major follow-up study (Maden et al, 1999) was based on the Dennis Hill Unit, which largely concentrated on rehabilitating patients from high security. Arnold Lodge itself was also unique among medium secure units in that it provided a dedicated treatment service for individuals with a diagnosis of personality disorder (McMurran et al, 1998). Although this is important in providing information on the course of such individuals after discharge, the impact of this group is unlikely to feature in other units. An additional limitation, as with any long-term follow-up, is that these outcome data reflect the therapeutic practice and policies of Arnold Lodge at the time; these have changed substantially over 20 years. Most importantly, the major factor influencing longterm outcome is likely to be the care patients received after discharge. This varied geographically across the region, from well-established community forensic services to poorly developed services relying on a series of locum consultants. There have also been major changes over time in treatment: for example the use of atypical antipsychotic medication and the availability of assertive outreach services, which might be expected to have a positive impact on an individual's course, were only in evidence towards the end of the study.
However, these are criticisms that can be levelled at any long-term follow-up study (Stone, 1990).

While acknowledging these limitations, it is also important to point to a major advantage of the study. Section 60 approval meant that patient ascertainment was high, as patients' consent to follow-up was not required. This is important in a group containing a large number of individuals with antisocial tendencies, where it is generally accepted that it is difficult to obtain such consent (Paris, 2003), and that if there is an ascertainment bias those who consent to be studied are likely to have the better outcome. The use of multiple data sources in our study also improved accuracy and reduced attrition ( Friendship et al, 2001; Francis et al, 2002).

\section{Mortality}

One of the most striking findings of this study was that a six-fold increase in mortality compared with the general population compares unfavourably with rates reported for other psychiatric groups. For instance, Harris \& Barraclough (1998) in a major review reported SMRs of 156 and 141 respectively for men and women with schizophrenia and 184 for those with personality disorder. Similarly, a general psychiatry first admission sample (using a similar method of recruitment to our study) had an SMR of 136, which was not significantly raised compared with the general population, and no suicide, despite an almost complete follow-up over 16 years (Naik et al, 1997). A study of deaths in 1996-1997 among ex-prisoners, offenders on community sentences and prisoners reported SMRs of 276, 358 and 150 (Sattar, 2001). Thus, our sample had a significantly greater mortality than other psychiatric and criminal justice samples, and it may therefore be a genuinely 'high-risk' population in terms of suicide and unnatural death (for further discussion see Davies et al, 2001).

Death, particularly from suicide, represents the end-point of a number of complex and long-term processes. Following their first admission to a medium secure unit patients will have had a variety of experiences; many will have continued to receive psychiatric services; and some will have remained as in-patients over the entire period of follow-up. Some patients will have returned to the criminal justice system with no further psychiatric contact; others will have been discharged or lost to follow-up in the community. The vast majority will continue to experience mental disorder with its long-term risk of suicide and increased mortality. In addition these people will carry the stigma of previous offending, and many will be convicted of further offences; to this will be added further risk factors such as difficulties in obtaining employment, finding accommodation and maintaining social networks, resulting in poverty and social exclusion. Factors detrimental to physical health such as obesity, lack of exercise, smoking and the sideeffects of antipsychotic medication (such as diabetes and cardiac arrhythmias) are also common in psychiatric populations. The message for general psychiatric services that will in the main be responsible for such patients is that this population's risks of mortality are high, probably related to psychiatric illness, treatment and lifestyle, and that all of these problems need to be addressed, as well as risks to others.

\section{Reconviction}

A methodological strength of our study was the use of multiple sources to minimise attrition and corroborate conviction data. Although the rates of conviction could be considered to be high, they are less than those found in other criminological samples. For instance, about a quarter $(26 \%)$ of this sample were convicted of a standard list offence within 2 years of discharge (or $30 \%$ of those discharged directly to the community), compared with the $58 \%$ of prisoners released in 2001 who were reconvicted of a standard list offence within 2 years (Home Office, 2002). Reducing reoffending is difficult as the criminal justice system has discovered, with several initiatives and legislation only succeeding in reducing reoffending in England and Wales by $1.3 \%$ between 1997 and 2001 (Home Office, 2006). The lowest rates of reconviction are for those with the longest periods of detention and closest supervision, namely life-sentenced prisoners and restricted patients. The reconviction rates for standard list and grave offences at 5 years (for those with previous convictions) are $17 \%$ and $3 \%$ for restricted patients and $10 \%$ and $1 \%$ for life licences respectively (Kershaw et al, 1997). However, many discharged patients were also involved in violent incidents or acts of arson ( $42 \%$ and $6 \%$ respectively at 5 years) for which they were not charged or convicted. 
An important outcome for those who provide community psychiatric services is the number of serious offences - especially homicide, inquiries following which have done so much to shape mental health policy. In this follow-up, five men were convicted of manslaughter during the study period, with one being convicted of two counts of attempted murder in addition to his conviction of manslaughter. All five offences took place in the community. Four patients had a Mental Health Act classification of mental illness and one had a classification of mental illness and psychopathic disorders. The duration from discharge to the offence varied from 3 months to over 9 years.

Although the outcome for reoffending was generally poor, there were two positive findings. The first was that although there is evidence that the level of risk among those admitted to medium secure units has increased, there has not been an increase in the rate of reconviction over time. Reconviction rates were analysed comparing patients discharged during the first 10 years of the study with those discharged during the last 10 years of the study: there was no significant difference in the numbers of patients reconvicted of standard list or grave offences at 2 years or 5 years. The second encouraging finding, in contrast to previous studies (e.g. Steels et al, 1998), was that those with a classification of psychopathic disorder did not have a significantly higher rate of reconviction than those with a classification of mental illness.

\section{Employment}

Employment is an important and tangible factor in the quality of living. Mentally disordered offenders are doubly censured, as both mental disorder and criminal history can be barriers to gaining employment. Patients who enter medium secure care often have limited experience of employment and a lower than average standard of education. Our finding that patients with a Mental Health Act classification of psychopathic disorder were more likely than patients with a classification of mental illness to have gained employment at some point during the follow-up is in line with previous research (Steels et al, 1998).

STEFFAN DAVIES, MRCPsych, Arnold Lodge, East Midlands Centre for Forensic Mental Health, Leicester; MARTIN CLARKE, BSc, Department of Health Sciences, University of Leicester, and Arnold Lodge, East Midlands Centre for Forensic Mental Health, Leicester; CLIVE HOLLIN, PhD, Department of Psychology, University of Leicester; CONOR DUGGAN, BSc, PhD, MD, FRCPsych, Division of Forensic Mental Health, University of Nottingham, Arnold Lodge and East Midlands Centre for Forensic Mental Health, Leicester, UK

Correspondence: Professor Conor Duggan, Division of Forensic Mental Health, University of Nottingham, Arnold Lodge, East Midlands Centre for Forensic Mental Health, Cordelia Close, Leicester, LE5 OLE, UK. Email: conor.duggan@nottshc.nhs.uk

(First received 28 July 2006, final revision 19 December 2006, accepted 19 January 2007)

\section{Implications of the study}

Overall the long-term outcome for former patients from the medium secure unit in this study was poor, with excess mortality, high rates of reconviction and readmission, and few gaining employment. Advances in mental health provision will, we hope, have a positive impact on an individual's course in future years. For community services, particularly assertive outreach, community forensic and community mental health teams, the message must be that risk in terms of mortality, morbidity and harm to others remains high in this population over long periods. Follow-up care needs to be consistent and long-term, and information on risk should not be lost or overlooked an increasingly difficult task with the multiplicity of teams and continual reorganisation of psychiatric services.

\section{ACKNOWLEDGEMENTS}

The initial description of the cohort (Ricketts et al, 200I) and follow-up using Office for National Statistics data were funded by Nottinghamshire Healthcare NHS Trust; further follow-up was funded by the NHS National Forensic Mental Health Research and Development Programme. The study took place at the Arnold Lodge medium secure unit; the continued support of this unit is gratefully acknowledged. We thank Dr Martin Tobin, Senior Lecturer in Genetic Epidemiology, University of Leicester, for advice on standardised mortality ratios, and Richard Twiner and Emma Booker for assisting in data collection.

\section{REFERENCES}

Davies, S., Naik, P. \& Lee, A. S. (200I) Depression, suicide and the national service framework. BMJ, 322 I500-150|.

Francis, B., Crosland, P. \& Harman, J. (2002) The Police National Computer and the Offenders Index: can they be combined for research purposes? Home Office
Research Findings 170. Home Office Research, Development and Statistics Directorate.

Friendship, C., Thornton, D., Erikson, M., et al (2001) Reconviction: a critique and comparison of two main data sources in England and Wales. Legal and Criminological Psychology, 6, 121-129.

Harris, E. C. \& Barraclough, B. (1998) Excess mortality of mental disorder. British Journal of Psychiatry, 173, II-53.

Home Office (2002) Criminal Statistics, England and Wales 200I. Cm 5696. TSO (The Stationery Office).

Home Office (2006) A Five-Year Strategy for Protecting the Public and Reducing Offending. TSO (The Stationery Office).

Kershaw, C., Dodswell, P. \& Goodman, J. (1997) Restricted Patients: Reconvictions and Recalls by the End of 1995: England and Wales. Home Office Research, Development and Statistics Directorate.

Maden, A., Rutter, S., McClintock, T., et al (1999) Outcome of admission to a medium secure psychiatric unit: I. Short- and long-term outcome. British Journal of Psychiatry, 175, 313-316.

Maden, A., Scott, F., Burdett, R., et al (2004) Offending in psychiatric patients after discharge from medium secure units: prospective national cohort study. $B M]$, 328, I534.

McMurran, M., Egan, V., \& Ahmadi, S. (1998)

A retrospective evaluation of a therapeutic community for mentally disordered offenders. Journal of Forensic Psychiatry, 9, 103-114.

Naik, P. C., Davies, S., Buckley, A. M., et al (1997) Long-term mortality after first psychiatric admission. British Journal of Psychiatry, 170, 43-46.

Paris, J. (2003) Personality Disorders Over Time: Precursors, Course and Outcome. American Psychiatric Publishing.

Ricketts, D., Carnell, H., Davies, S., et al (200I) First admissions to a regional secure unit over a 16-year period: changes in demographic and service characteristics. Journal of Forensic Psychiatry, 12, 78-89.

Sattar, G. (200I) Rates and Causes of Death Amongst Prisoners and Offenders under Community Supervision. Home Office Research Study 231. Home Office Research, Development and Statistics Directorate.

Steels, M., Roney, G., Larkin, E., et al (1998) Discharged from special hospital under restrictions: a comparison of the fates of psychopaths and the mentally ill. Criminal Behaviour and Mental Health, 8, 39-55.

Stone, M. (1990) The Fate of Borderline Patients: Successful Outcome and Psychiatric Practice. Guilford. 